\title{
Curcumin induces apoptosis in trophoblast model cell line
}

\section{Tatit Nurseta, Yahya I, I W.A Wiyasa, Rahajeng, Imelda, Putu A.R. Ratnawati}

Department of Obstetrics and Gynecology, Saiful Anwar General Hospital, Faculty of Medicine, Universitas Brawijaya, Malang, Indonesia

\section{ABSTRACT}

Background: Several studies have reported that curcumin exerts chemopreventive effects in various type of cancers, through several mechanisms, however, the effect of curcumin on carcinogenesis in patients with hydatidiform mole has not yet been investigated. This study was conducted to evaluate the effect of curcumin on apoptosis, proliferation, and nuclear translocation of endothelial nitricoxide synthase in trophoblast cells induced by estradiol in complete hydatidiform mole (CHM).

Methods: In this in vitro study, trophoblast cells were divided into six groups, the control group (trophoblast cells were exposed to $100 \mathrm{pg} / \mathrm{mL}$ of $17-\beta$ estradiol) and the treatment group (trophoblast cells were exposed to $100 \mathrm{pg} / \mathrm{mL}$ of $17-\beta$ estradiol in the presence of curcumin with doses: 50, 100, 200, 400, and $800 \mu \mathrm{M}$ ). At the end of study, the cell proliferation was analyzed using MTT assay and apoptosis with TUNEL test in each group thropoblast cell. eNOS translocation was assayed using confocal laser scanning microscopy at the various dose of curcumin.

Results: Curcumin at the doses of 200, 400, and $800 \mu \mathrm{M}$ significantly decreased the proliferation and increased the apoptotic index in curcumin-treated group compared to those in the control group $(\mathrm{p}<0.05)$. All doses of curcumin treatment significantly decreased the nuclear eNOS expression compared to that in the control group. The three highest doses of curcumin increased cytoplasmic eNOS expression compared to that in control group.

Conclusion: Curcumin inhibits the proliferation and modulates the apoptosis of trophoblast cells induced by estradiol in CHM involvement.

Keywords: apoptosis, curcumin, eNOS, hydatidiform mole, trophoblast

pISSN: 0853-1773• eISSN: 2252-8083• https://doi.org/10.13181/mji.v27i2.1821 • Med J Indones. 2018;27:87-93

- Received 06 Feb 2017 • Accepted 27 Apr 2018 
Gestational trophoblastic disease (GTD) predominantly affects women and is characterized by neoplastic and non-neoplastic lesions from different types of villous and nonvillous trophoblasts. Hydatidiform mole is an overgrowth of villous trophoblasts and affects approximately 1 of 120-500 pregnancies in Asian countries. Based on their clinicopathological, karyotypic, and genetic analysis, hydatidiform moles have been categorized as complete moles and partial. CHM is characterized by gross hydropic swelling and variable trophoblastic proliferation involving the chorionic villi. ${ }^{1,2}$

Estrogen-activated receptors regulate gene transcription by directly binding to deoxyribose nucleic acid (DNA). This ligandreceptor interaction also drives cellular processes via "extra-nuclear" non-genomic effects. Two important pathways are switched on, i.e. the phosphatidylinositol 3-kinase (PI3K) and extracellular signal-regulated kinase $1 / 2$ (ERK1/2) [also known as p42/p44 mitogen activated protein kinase (MAPK)] pathways. ${ }^{3,4}$ This signal is important for the proliferation of cancer cells.

Nitric oxide (NO) is a short-lived, pleiotropic molecule generated by three isoforms of NO synthase (NOS) that plays complicated roles in tumor biology. ${ }^{5} \mathrm{NO}$ is synthesized from L-arginine and oxygen by four major isoforms of NO synthase (NOS): neuronal NOS, endothelial NOS (eNOS), inducible NOS (iNOS) and, more recently, mitochondrial NOS. eNOS can be activated by post-translational modification (e.g., phosphorylation by ERK1/2 or PI3K/Akt pathways) or by transcription (e.g., NF- $\kappa B$ ). Depending on the cellular milieu, release of low concentrations of NO can modulate cancer-related events such as angiogenesis, apoptosis, cell cycle, invasion, and metastasis. ${ }^{6-8} \mathrm{An}$ in vivo study shows that changes in eNOS levels play a pivotal role in eNOS in chronic stress-induced initiation and promotion of tumor growth. ${ }^{9}$ Previous studies have shown that eNOS was present in trophoblast cells of partial and complete hydatidiform moles and in choriocarcinoma. ${ }^{10}$ Estrogen activates eNOS via kinase activation pathway. ${ }^{11}$ Previous studies have demonstrated showed that NO and three NOS isoforms have dual roles, i.e., promoting cancer cell apoptosis and inhibiting cancer cell apoptosis. According to these studies, high eNOS expression may be cytotoxic to tumor cells, whereas low of eNOS expression may have an opposite effect of enhancing the tumor growth. ${ }^{12,13}$ These data indicate that there is a relationship between the proliferative effect of estrogen with eNOS expression in cancer cells.

Curcumin is an active natural polyphenol derived from the root of Curcuma longa L. and has been considered as a great pharmacological agent due to its safety and effectiveness in cancer treatment. ${ }^{14}$ Curcumin stimulates apoptosis of tumor cell or inhibits the cell proliferation, angiogenesis, and metastasis. It also has different properties such as anti-inflammatory, antioxidant, antimicrobial and wound healing promoting agent. ${ }^{15,16}$ The effects of curcumin are generally due to the interactions between curcumin and multiple cell-surface receptors or cytokines. ${ }^{17}$ Till date, no study has evaluated the effects of curcumin on the trophoblast cells of complete hydatidiform mole. Therefore, this study was conducted to investigate whether curcumin modulates the proliferation, apoptosis, and the characteristics of eNOS translocation in the trophoblast cells of a complete hydatidiform mole.

\section{METHODS}

This study was conducted at the Laboratory of Cell Culture/Tissue of Faal Laboratory, Faculty of Medicine, Universitas Brawijaya Malang from June to November 2013. The study design was simple, non-blinded experimental with the analysis performed at the end of treatment (post-test control group design). This study was approved by the Health Research Ethics Committee of the Faculty of Medicine of Universitas Brawijaya, Number 400/ LXIII/K.3/302/2013.

\section{Materials}

Human trophoblast cells of a complete hydatidiform mole were obtained from a woman who met the following criteria: patient with a complete hydatidiform mole undergoing curettage agreeing to sign the informed consent.

\section{Cell culture}

This in vitro study was conducted using the culture of complete molar pregnancy trophoblast cells from an complete hydatidiform mole (CHM) 
patient undergoing curettage. Complete molar pregnancy cell cultures were exposed to $17 \mu \mathrm{g}$ estradiol at a dose of $100 \mu \mathrm{g} / \mathrm{ml}$, perform exposed with various doses of curcumin and no exposure, the proliferation index was evaluated using the 3-[4,5-dimethylthiazol-2-yl]-2,5-diphenyl tetrazolium bromide (MTT) method and apoptotic index by terminal deoxynucleotidyltransferasemediated nick-end labeling (TUNEL) DNA fragmentation labeling method. The apoptotic index was evaluated using the TUNEL immunohistochemical system, in which the nucleus of the cells undergoing apoptosis exhibits DNA fragmentation, and by the TUNEL method will be detects enzymatically and imparts a brown color to the nucleus of apoptotic cells.

Immediately after curettage, the complete hydatidiform mole was washed using normal saline and placed in a cord solution obtained from newborn bovine serum (NBS), which was collected from a calf that was less than 10 days old and maintained under cold condition until the isolation of trophoblast cells. The trophoblast cells were isolated before $12 \mathrm{~h}$ after curettage. The trophoblast cells was obtained from the mole tissue were washed with NS to separate from erythrocytes and washed using media (M199 (medium) + Pen Strep (antibiotics) + L-glutamine $+\mathrm{Na}_{2} \mathrm{CO}_{3}$ ) incubated solution of collagenase (type I) $7 \mathrm{mg} / 10 \mathrm{cc}$ for $2 \mathrm{~h}$ in a centrifuge tube, a medium in waterbath shaker $37^{\circ} \mathrm{C}$, centrifuged at $2000 \mathrm{rpm}$ for $7 \mathrm{~min}$, produce supernatant and pellet, supernatant removed pellet was given medium, re-homogenized, the second supernatant and pellet, supernatant was removed then pellets added media + fetal bovine serum (FBS), incubated in incubators $37^{\circ} \mathrm{C}$ and $5 \% \mathrm{CO}_{2}$, observed under microscope (until cell grow), perform $\beta$-human chorionic gonadotropin (HCG) test. This cell suspension was seeded into culture wells that had been coated with gelatin. After reaching confluency, the cells were divided into six replicates, including a control group (trophoblast cells exposed to $100 \mathrm{pg} / \mathrm{ml}$ of $17-\beta$ estradiol without exposure to curcumin) and a treatment group (trophoblast cells exposed to $100 \mathrm{pg} / \mathrm{ml}$ of $17-\beta$ estradiol in the presence of curcumin at the following five doses: 50,100 , 200,400 , and $800 \mu \mathrm{M}$. The curcumin doses were prepeared using $1 \mathrm{gr}$ of curcumin powder and measured using an analytical balance to prepare a different concentration.

\section{Analysis of cell proliferation}

In vitro cytotoxicity of curcumin against the trophoblast cells of the complete hydatidiform mole was evaluated using MTT assay. ${ }^{18}$ The trophoblast cells were seeded at a density of $2 \times 10^{3}$ cells in 96-well plates and incubated overnight. Subsequently, the cells were exposed to a series of different curcumin concentrations. This was followed by incubation of the cells with treatment for 24 and $48 \mathrm{~h}$. At the end of this period, the percentage of viable cells relative to the untreated control was determined on the basis of the mitochondrial conversion of MTT to formazan. The results were expressed from four measurements as mean \pm standard deviation (SD).

\section{Analysis of cell apoptosis}

Following the manufacturer's protocol, TUNEL staining was conducted using an in situ cell death detection kit (Dead End TM Fluorometric TUNEL System, Promega, Madison, USA) to assess the apoptotic cells in the tumor. TUNEL staining is based on the enzymatic addition of digoxigenin-nucleotide to the incised DNA by recombinant terminal deoxynucleotidyl transferase (rTdT). All the five tumor cells in each group were sectioned in the TUNEL staining tests. In the tumor tissue sections, five equalsized areas were randomly chosen and analyzed. Then, the apoptotic index was calculated based on the number of the TUNEL-positive cells or the total number of cells in the five randomly selected tumor areas in each tumor sample. This analysis was conducted by two independent investigators in a blinded manner. ${ }^{19}$

\section{Immunofluorescence assay}

Immunofluorescence staining was conducted to determine the eNOS expression in the trophoblast cells. The slides were incubated with primary anti eNOS rabbit antibody (1:1000 dilution; Santa Cruz Biotechnology Inc., USA) and mouse antirabbit IgG-FITC (1:1500; Santa Cruz Biotechnology Inc., USA) for 1 hour each in dark conditions. The visualization and measurement of the eNOS expression were conducted using confocal laser scanning microscopy (CLSM; Olympus Corporation, Tokyo, Japan). Three areas of the slides were examined. The cytoplasmic and nuclear eNOS expressions were calculated using the FluoView software, version 1.7a (Olympus Corporation, Tokyo, Japan). 


\section{Statistical analysis}

Data presented using mean value of \pm SD and differences between the groups were analyzed by one-way analysis of variance (ANOVA) using SPSS 17.0 statistical package. The significant results of ANOVA were tested using the post hoc test in which $\mathrm{P}<0.05$ was considered to be statistically significant.

\section{RESULTS}

Figure 1A shows the histogram of mean \pm SD of the proliferation index of trophoblast model cells in the six groups of curcumin dose 50, 100, 200, 400, and $800 \mu \mathrm{M}$ ). Based on one-way ANOVA analysis, the proliferation index significantly decreased at all doses of curcumin compared to the untreated control group ( $\mathrm{p}<0.05)$. Continued with least significant difference (LSD) analysis, which is the multiple comparison test, obtained the proliferation index of trophoblast significantly decreased at three highest doses of curcumin and have a same ability to decrease the proliferation index, compared to $50 \mu \mathrm{M}$ curcurmin dose and control group statistically.

Figure 1B shows the histogram of mean \pm SD of the apoptotic index in the curcumintreated group $(50 \mu \mathrm{M}, 100 \mu \mathrm{M}, 200 \mu \mathrm{M}, 400$ $\mu \mathrm{M}$, and $800 \mu \mathrm{M})$. Based on one-way ANOVA analysis, the apoptosis index significantly increased at all doses of curcumin compared to the untreated control group $(\mathrm{p}<0.05)$. Same as the proliferation index, data analysis continued with least significant difference (LSD) analysis, obtained the proliferation index of trophoblast significantly decreased at three highest doses of curcumin and have a same ability to decrease the proliferation index, compared to $50 \mu \mathrm{M}$ curcurmin dose and control group. Cell apoptosis index was highest at $400 \mu \mathrm{M}$, thus a dose of $400 \mu \mathrm{M}$ can be considered the most optimal dose of curcumin in increasing the apoptotis index.

The eNOS expression was found to vary in each group. In control group there was activation of eNOS translocation from the cytoplasm to the nucleus. The expression of eNOS is denoted by the amount of green dots displayed after imunofluoresence staining. The more the eNOS expression is activated, the more
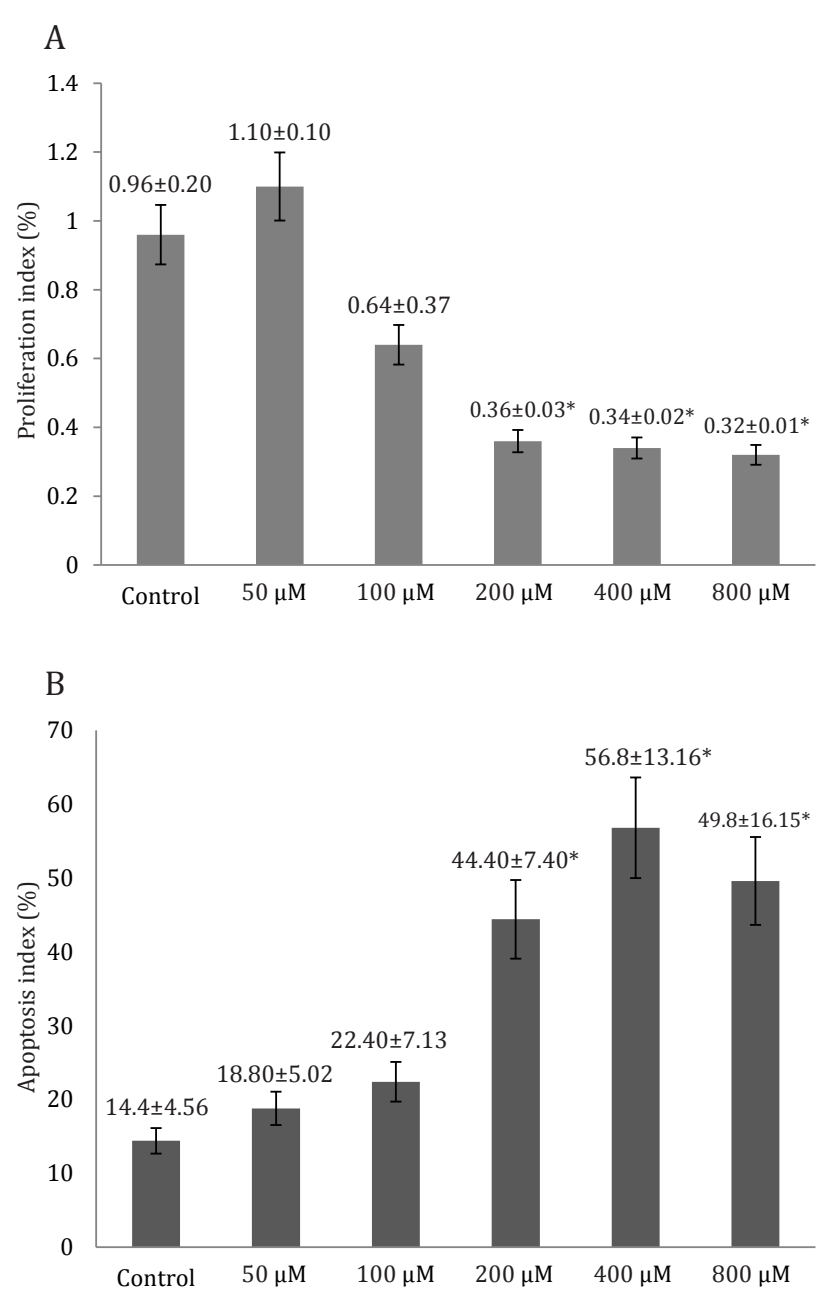

Figure 1. A) Proliferation index of trophoblast significantly decreased at all doses of curcumin $(p=0.001)$, based on oneway Anova analysis compared to control group. B) Apoptosis index of trophoblast significantly increased at all doses of curcumin ( $\mathrm{p}=0.001$ ), based on one-way Anova and LSD post hoc analysis compared to control group ${ }^{*} \mathrm{p}=<0.005$

greener or brighter or the more amount of green dots are displayed.

Figure 2 shows that the tendency of eNOS activation in the nucleus is higher than that in the cytoplasm (Fig. A). The expression of eNOS at the $50 \mu \mathrm{M}$ dose of curcumin in the nucleus was lower than that in the cytoplasm (Fig. B), whichindicated that the $50 \mu \mathrm{M}$ dose of curcumin could deacrease the eNOS translocation from the cytoplasm to the nucleus in complete hydatidiform mole. In the treatment group, the dose of $100 \mu \mathrm{M}$ curcumin also decreased the activation of eNOS in the nucleus compared to control group (Fig. C). Interestingly, there was an increasing trend of eNOS activation in the nucleus at the curcumin dose of 200,400 , and $800 \mu \mathrm{M}$ (Fig. D,E,F). 

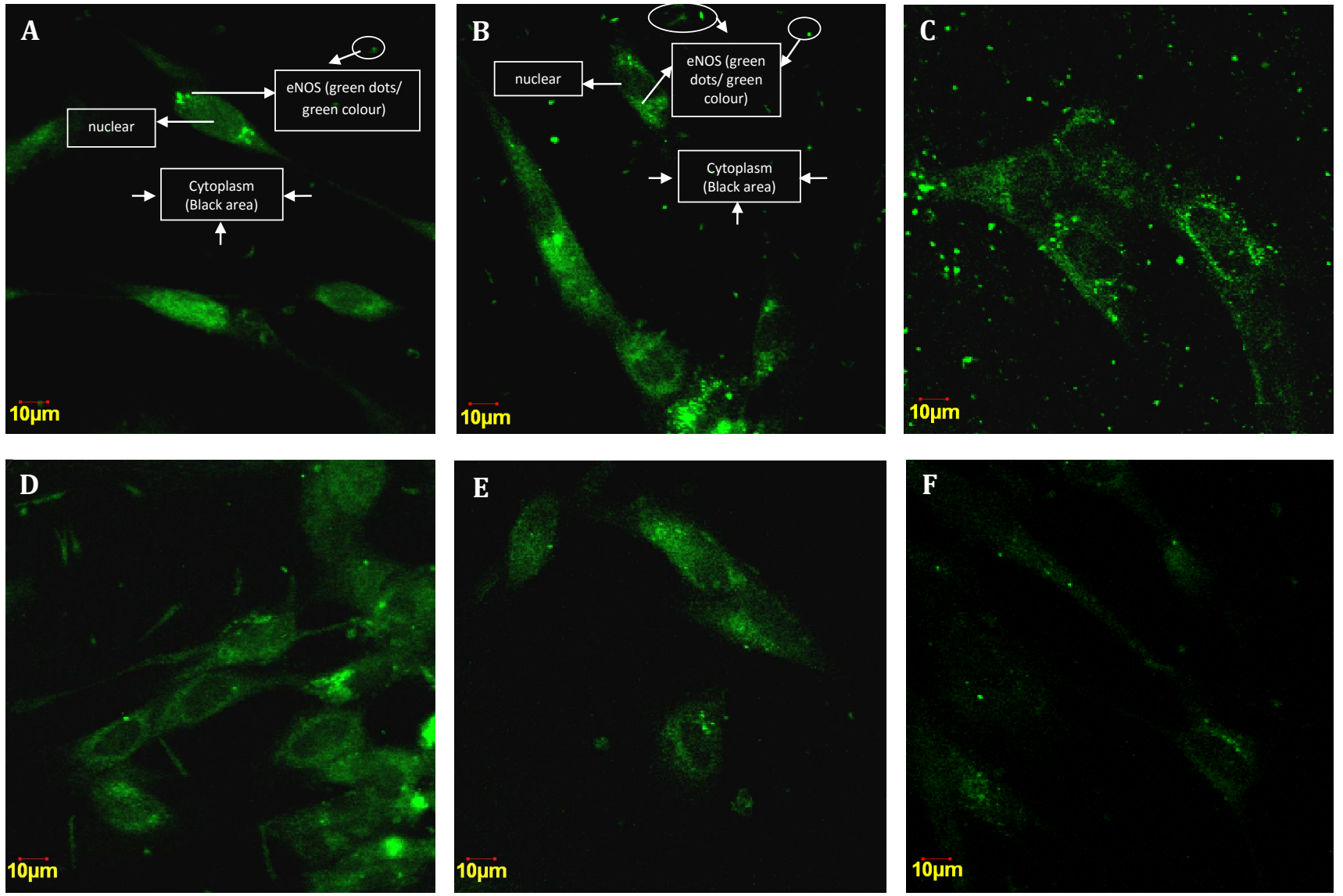

Figure 2. Cytoplasmic and nuclear eNOS expression in the trophoblast cell culture of complete hydatidiformmole. A) Imunofluoresencestaining in control group; B) Imunofluoresence staining in $50 \mu \mathrm{M}$ group; C) $100 \mu \mathrm{M}$ group; D) $200 \mu \mathrm{M}$; E) $400 \mu \mathrm{M}$; F) $800 \mu \mathrm{M}$

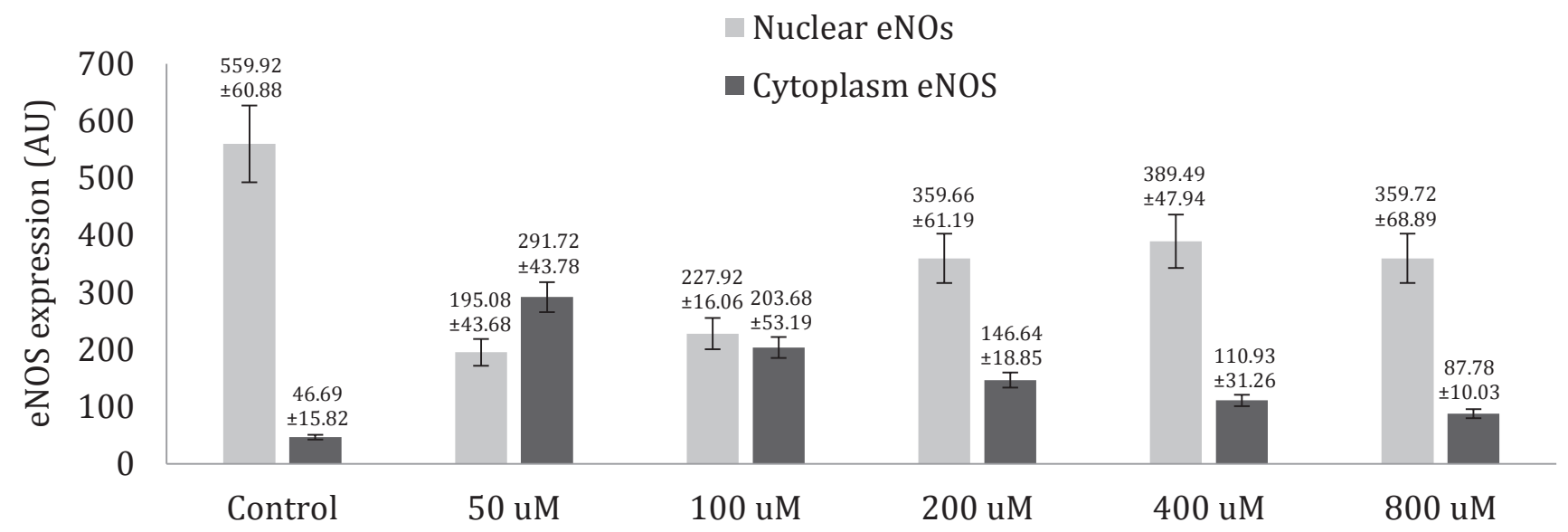

Figure 3. eNOS activation in nuclear and cytoplasm significantly difference based on one-way ANOVA analysis compared to control group $(\mathrm{p}<0.05)$.

Based on one-way ANOVA results, eNOS activation is significantly difference in nucleus and cytoplasm at all doses of curcumin compared to control group $(\mathrm{p}<0.001)$. Furthermore, multiple comparison tests using LSD showed that the mean eNOS activation in nucleus was significantly different in the trophoblast model cellswhen compared betweenthe control group and the groups treated with curcumin doses of $50,100,200,400$, and $800 \mu \mathrm{M}$ dose. The mean 
eNOS activation in the nucleus was lowest at 50 and $100 \mu \mathrm{M}$ curcumin doses (Figure 3).

The result of multiple comparison test using the LSD showed that eNOS activation in the cytoplasm was significantly different at the curcumin doses of 50,100, 200 and $400 \mu \mathrm{M}$. However, there was no significant difference in the mean eNOS activation at $800 \mu \mathrm{M}$ curcumin dose compared to that in the control group. Thus, treatment with $50,100,200$, and $400 \mu \mathrm{M}$ curcumin doses in trophoblast model cell cultures can increase eNOS activation in the cytoplasm. This was indicated by a significant increase in mean eNOS activation rates in the treatment group when compared to that in the control group, except at dose $800 \mu \mathrm{M}$ (Figure 3).

\section{DISCUSSION}

Curcumin (diferuloylmethane), a yellow pigment present in the rhizomes of turmeric, is used in cooking in India and Indonesia. ${ }^{20}$ Curcumin regulates the tumor cell growth through multiple cell signaling pathways, an antiproliferative agent, a survival signal reduction, induces programmed cell (apoptosis) to mitochondria and caspases activation. ${ }^{21}$ Estrogen-activated receptors regulate gene transcription by directly binding to DNA.

In this study, only three highest curcumin doses significantly decreased the proliferation index in the curcumin-treated group compared to that in the untreated control group $(\mathrm{p}<0.05)$, which could regulate tumor cell growth through cell proliferation pathway. ${ }^{18}$ It is hypothesized that an antiproliferative effect of curcumin on the trophoblast cells of the complete hydatidiform mole could be derived from the inhibition of nuclear factor of kappa-light-chain-enhancer of activated B cells (NF- $\kappa B$ ).NF- $\kappa B$ acts as an oncogene through its ability to stimulate cell proliferation and survival that which can be reduced by curcumin. ${ }^{20,21}$

Apoptosis is an active and a physiological mode of cell death that is commonly restored by anticancer agents. ${ }^{22}$ The results of this study also showed that curcumin treatment can regulate the growth of the complete hydatidiform mole through the cell apoptosis pathway. These results were consistent with those of previous studies showing that curcumin inhibits the anti-apoptotic protein and regulates the pro-apoptotic protein. ${ }^{23}$ The inhibitors of NF- $\kappa \mathrm{B}$ expose the cancer cells to apoptosis. ${ }^{24} \mathrm{~A}$ previous study reported that the administration of $100 \mathrm{pg}$ of 17- $\beta$ estradiol could increase NF- $\kappa \mathrm{B}$ expression in $\mathrm{CMH}$ trophoblast cells cultures. Extensive research has indicated that this $N F-\kappa B$ plays an important role in enhancing cell proliferation, among other function, by increasing the expression of cyclin-D1, c-myc, c-erb, c-fms, MMP-9, growth factors, and p53, and it is also involved in carcinogenesis processes. Curcumin can decrease cell proliferation directly through its effect on the target molecule, as well as through resistance to NF- $\mathrm{KB}$.

eNOS and its participation in combinatorial complexes with Estrogen Receptor Beta (ER $\beta$ ) and Hypoxia Inducible Factors (HIFs) that determine localized chromatin remodeling in response to estrogen (E2) and hypoxia stimuli, resulting in transcriptional regulation of genes associated with adverse prognosis target genes. ${ }^{25}$ Moreover, estrogen can active eNOS via kinase pathway. ${ }^{11}$

In this study, all doses of curcumin treatment significantly decreased the nuclear eNOS expression compared to that in the untreated control group $(\mathrm{p}<0.05)$. Interestingly, the administration of $200,400 \mu \mathrm{M}$, and $800 \mu \mathrm{M}$ curcumin to the estrogen-treated trophoblast cells of the complete hydatidiform mole increased the cytoplasmic eNOS expression compared to that in the untreated group. This finding indicated that the two lowest doses of curcumin significantly inhibited eNOS translocation from the cytoplasm to the nucleus of complete hydatidiform mole. It is hypothesized at the three highest doses of curcumin, the localization of eNOS is involved in the proliferation and apoptosis of trophoblast cells of the complete hydatidiform mole. It corresponds with a previous study that showed that curcumin affects nuclear apoptosis and proliferation. $^{21}$

This study has limitations that require an advanced research to investigate the effect of curcumin in activating the apoptosis cascade, both extrinsic and intrinsic. Clinical trial are needed to confirm the effect of curcumin in patients with a hydatidiform mole. The findings of this study are important, indicating that curcumin can be used as a complementary therapy in women affected 
with complete hydatidiform moles and also as a chemopreventive agent.

In conclusion, the high doses of curcumin inhibited the proliferation and modulated the apoptosis of trophoblast cells induced by estradiol in the complete hydatidiform mole through the localization of eNOS.

\section{Conflicts of Interest}

The authors affirm no conflict of interest

in this study.

\section{Acknowledgement}

The authors acknowledged all the technicians in the Laboratory of Physiology Molecular for helping in this study.

\section{REFERENCES}

1. Strohl A, Lurain J. Clinical epidemiology of gestational trophoblastic disease. Curr Obstet Gynecol Rep. 2014;3:40-3.

2. Chen KH, Hsu SC, Chen HY, Ng KF, Chen TC. Utility of fluorescence in situ hybridization for ploidy and p57 immunostaining in discriminating hydatidiform moles. Biochem Biophys Res Comm. 2014;446:555-60.

3. Shupnik MA.Crosstalk between steroid receptors and the c-Src-receptor tyrosine kinase pathways: implications for cell proliferation. Oncogene. 2004;23(48):7979-89.

4. Cheskis BJ, Greger J, Cooch N, McNally C, McLarney $\mathrm{S}$, Lam HS, et al. MNAR plays an important role in ERa activation of Src/MAPK and PI3K/Akt signaling pathways. Steroids. 2008;73(9-10):901-15.

5. Burke AJ, Sullivan FJ, Giles FJ, Glynn SA. The yin and yang of nitric oxide in cancer progression. Carcinogenesis. 2013;34(3):503-12.

6. Ying L, Hofseth LJ. An Emerging role for endothelial nitric oxide synthase in chronic inflammation and cancer. Cancer Res. 2007;67(4):1407-10.

7. Xun W, Zhi FW, Yin X, Rui R, Bao LH, Ze XS. Association between three eNOS polymorphisms and cancer risk: a meta-analysis. Asian Pac J Cancer Prev. 2014;15(13):5317-24.

8. Temiz C, Arslan M, Sayin M, Vatansever M, Giray G, Va A. Effects of nitric oxide-vascular endothelial growth factor systems in chick embryo cerebral vasculogenesis and angiogenesis. J Clin Anal Med. 2012;3(4):393-7.

9. Barbieri A, Palma G, Rosati A, Giudice A, Falco A, Petrillo A, et al. Role of endothelial nitric oxide synthase (eNOS) in chronic stress-promoted tumour growth. J Cell Mol Med. 2012;16(4):920-6.

10. Barut A, Harma M, Arikan I, Barut F. Endothelial nitric oxide synthase expression in gestational trophoblastic diseases. Int J Gynecol Cancer. 2010; 20(3):337-40.

11. Lu Q Schnitzler GR, Ueda K, Iyer LK, Diomede OI, Andrade T, et al. ER alpha rapid signaling is required for estrogen induced proliferation and migration of vascular endothelial cells. PLoS ONE. 2016;11(4): e0152807.

12. Vanini F, Kashfi K, Nath N. The dual role of iNOS in cancer. Redox Biol. 2015;6:334-43.

13. Zhou L, Wang Y, Tian D, Yang J, Yang Z. Decreased levels of nitric oxide production and nitric oxide synthase- 2 expression are associated with the development and metastasis of hepatocellular carcinoma. Mol Med Rep. 2012;6(6):1261-6.

14. Bandyopadhyay D. Farmer to pharmacist: curcumin as an anti-invasive and antimetastatic agent for the treatment of cancer. Front Chem. 2014;2:113.

15. Wilken R, Veena MS, Wang MB, Srivatsan ES. Curcumin: a review of anti-cancer properties and therapeutic activity in head and neck squamous cell carcinoma. Mol Cancer. 2011;10:12.

16. Noorafshan A, Ashkani-Esfahani S. A review of therapeutic effects of curcumin. Curr Pharm Des. 2013;19:2032-46.

17. Abuelba H, Cotrutz CE, Stoica BA, Stoica L, Olinici D, Petreus T. In vitro evaluation of curcumin effects on breat adenocarcinoma 2D and 3D cultures. Rom J Morphol Embryol. 2015; 56(1):71-6.

18. Jamalzadeh L, Ghafoori H, Sariri R, Rabati H, Nasirzade J, Hasani H. Cytotoxic effects of some common organic solvent on MCF-7, RAW-264.7 and human umbilical vein endothelial cells. Avicenna J Med Biochem. 2015;4(1):e33453.

19. Acharya S, Reddy R, Kuppili, Chaganti KL, Bose K. Proteases in apoptosis: protocols and methods. in: bose $\mathrm{k}$, editor.proteases in apoptosis: pathways, protocols and translational. 1st ed. Switzerland; Springer International Publishing; 2015. p. 143-202.

20. Rajesh E, Sankari LS, Malathi M, Krupaa JR. Naturally occurring products in cancer therapy. J Pharm Bioallied Sci. 2015;7(Suppl 1):S181-S3.

21. Park W, Amin AR, Chen ZG, Shin DM. New perspectives of curcumin in cancer prevention. Cancer Prev Res (Phila). 2013;6(5):387-400.

22. Du Y, Feng J, Wang RM, Zhang H, Liu J. Effects of flavonoids from Potamogeton crispus L. on proliferation, migration, and invasion of human ovarian cancer cells. PLoS One. 2015; 10(6): e0130685.

23. Mahmud M, Diwoni A, Filiczak N, Janicka M, Gubernatus J. Long-circulating curcumin-loaded liposome formulations with high incorporation effeciency, stability and anticancer activity towards pancreatic adenocarcinoma cell lines in vitro. PLoS One. 2016. 10:1371.

24. Notarbartolo M, Poma P, Perri D, Dusonchet L, Cervello M, D'Alessandro N. Antitumor effects of curcumin, alone or in combination with cisplatin or doxorubicin, on human hepatic cancer cells. Analysis of their possible relationship to changes in nuclear factor-kappaB (NF$\mathrm{kB}$ ) activation levels and in inhibitory of apoptosis proteins (IAP) gene expression. Cancer Letters. 2005;224(1):53-65.

25. Nanni S, Aiello A, Re A, Guffanti A, Benvenuti V, Collusi C. Estrogen-dependent dynamic profile of eNOSDNA assosiations in prostate cancer. PLoS One. 2013. 10:371-79. 